\title{
Sustainable operations: The cutting stock problem with usable leftovers from a sustainable perspective
}

\author{
Karen Rocha Coelho a , Adriana Cristina Cherri b, **, Edméa Cassia Baptista ${ }^{\text {b }}$, \\ Charbel José Chiappetta Jabbour ${ }^{\mathrm{c}, \mathrm{d}, *}$, Edilaine Martins Soler ${ }^{\mathrm{b}}$ \\ ${ }^{a}$ UNESP - Univ Estadual Paulista, Faculty of Engineering, Master's and PhD Program in Production Engineering, Avenida Eng. Edmundo C. Coube, 14-01, \\ Bauru, Sao Paulo State, 17033360, Brazil \\ ${ }^{\mathrm{b}}$ UNESP - Univ Estadual Paulista, Faculty of Science, Department of Mathematics, Avenida Eng. Edmundo C. Coube, 14-01, Bauru, Sao Paulo State, \\ 17033360, Brazil \\ ${ }^{c}$ Montpellier Business School, Montpellier Research in Management, 2300, Avenue des Moulins, 34185, Montpellier Cédex 4, France \\ ${ }^{\mathrm{d}}$ University of Stirling, Stirling Management School, Centre for Advanced Management Education, Scotland, FK9 4LA, United Kingdom
}

\section{A R T I C L E I N F O}

\section{Article history:}

Received 1 February 2017

Received in revised form

13 August 2017

Accepted 17 August 2017

Available online 29 August 2017

\section{Keywords:}

Cutting problem

Sustainable operations

Sustainable supply chain

Mathematical modelling

\begin{abstract}
A B S T R A C T
This paper proposes a mathematical model and two heuristic procedures to solve the cutting stock problem with usable leftovers, relating the implications of the model with aspects considering sustainability in terms of environmental, economic and social issues. The possibility of generating leftovers that can be used or sold, reduces raw material waste during the cutting process and, consequently, increases companies' profits. By reducing waste and increasing profits, companies can become more competitive in the market. They can also integrate environmental aspects into their operational strategies and, therefore, create a better self-image and profitability, generating more jobs and contributing to a stronger local economy. We believe that the model is more likely to be adopted by smaller companies, which generally face numerous barriers but at the same time have a significant social impact, generating income and jobs. Based on the knowledge of the authors, this is the first study that relates a cutting problem with its implications for sustainability. Computational tests were performed, and the obtained results are discussed considering the win-win approach to sustainability.
\end{abstract}

๑) 2017 Elsevier Ltd. All rights reserved.

\section{Introduction}

A positive link between operational research and sustainability has been proposed by various researchers around the world (Gonzalez et al., 2015; Gunasekaran and Irani, 2014), particularly concerning sustainable mathematical models (Brandenburg et al., 2014). A recent literature review showed that there are few studies that relationships classical problems of operational research and sustainability. This research gap was recently addressed by Jaehn (2016), who suggested, among others, that the cutting stock problem (CSP) and its relationship with sustainability

\footnotetext{
* Corresponding author. Montpellier Business School, Montpellier Research in Management, 2300, Avenue des Moulins, 34185, Montpellier Cédex 4, France.

** Corresponding author.

E-mail addresses: karenrc345@hotmail.com (K.R. Coelho), adriana@fc.unesp.br (A.C. Cherri), baptista@fc.unesp.br (E.C. Baptista), cjcjabbour@gmail.com (C.J. Chiappetta Jabbour), edilaine@fc.unesp.br (E.M. Soler).

as a subject needs to be developed further. This neglected gap in the literature was the foundation of this study.

The CSP consists of cutting a set of objects available in stock to produce a set of demanded items to meet the demands of customers while optimizing an objective function. This problem is essential for production planning in many industries such as paper, glass, steel bars and furniture, among others, and plays a crucial role in the current economy. These industries generally use large amounts of raw materials and there is often waste when items are cut. To minimize this negative effect, the cutting process needs to be planned. Minimizing the negative effects of the CSP can have a significant and positive effect on the corporate movement toward sustainable development.

Research on the CSP was first cited in 1939 with Kantorovich's study, which was published only in 1960. Gilmore and Gomory (1961) proposed the simplex method with column generation for a linear optimization model and solved an actual one-dimensional CSP for the first time. Gilmore and Gomory (1963) later presented a new method for the knapsack problem, which is a subproblem that arises when solving the CSP. 
Material leftovers are common during the cutting process, therefore some studies have investigated how to utilize these leftovers. This problem is a variation of the CSP and is known in the literature as the cutting stock problem with usable leftovers (CSPUL). The objective of the CSPUL is to produce a specific amount of items by cutting standard or retail objects (i.e., leftovers of previous cutting processes, according to Cherri et al., 2009), available in stock, minimizing waste material. Leftovers (retails) can be generated in quantities and limited lengths and be returned to the stock to meet future demands. Scheithauer (1991) presented a mathematical model for the one-dimensional CSPUL with the objective of minimizing leftovers, which was solved using the column generation technique proposed by Gilmore and Gomory (1963). The strategy used consists of including extra items (retails) in the problem without any demands to meet. Gradišar et al. (1997) presented a study about the CSPUL in the textile industry. In order to solve the problem, the authors proposed a mathematical model. However, to solve the problem, the authors followed a heuristic procedure.

Gradisar and Trkman (2005) limited the quantity of leftovers generated during the cutting process. A heuristic procedure was combined with a branch and bound method to solve the problem. Abuabara and Morabito (2009) used the mathematical model proposed by Gradišar et al. (1997) to solve the CSPUL in a Brazilian company that cuts structural metallic tubes used to build agricultural aircraft. To solve the CSPUL, Cherri et al. (2009) made changes to classical constructive and residual heuristics available in the literature to solve the CSP. Cui and Yang (2010) proposed an extension of the model by Scheithauer (1991). Cherri et al. (2013) modify some procedures proposed in Cherri et al. (2009) to consider a situation where retail items should not remain in stock for a long time. The performance of the proposed strategy was analyzed solving randomly generated dynamic instances with a time horizon. Cherri et al. (2014) presented a survey of existing papers that investigated the one-dimensional CSPUL.

Although there are some studies considering the CSPUL, the first one that proposes a mathematical model analyzing retail as part of production planning was presented by Arenales et al. (2015). In this study, a mathematical model was proposed considering that the lengths of the retails and their quantities are previously defined. The numerical tests performed showed that the proposed model performed well and there was a strong relationship between the amount of generated retail and waste reduction. Tomat and Gadišar (2017) considered the usable leftovers with consecutive demands. To avoid accumulation of retails in stock, the proposed method has the objective of finding the ideal quantity of retail items to stock and the ideal minimum length of new retail items generated.

Related to the two-dimensional problems, Andrade et al. (2013, 2016) proposed multilevel mixed integer programming (MIP) models to represent the two-dimensional cutting stock problem with usable leftovers. In these studies, there is no control on the number of leftover types.

The importance of the CSP and its variations for production planning can be observed by the number of papers presented in the literature. Considering the CSPUL, retails are generated simply to reduce waste and in all the approaches they are used internally. However, the possibility of also selling retails of stock to other industries that can use these objects as raw material in their production processes is not considered, although this is done on a regular basis in some small-sized companies to make better use of objects while maximizing profits. Furthermore, there is a significant gap in the literature on how CSPUL can be related to modern approaches of corporate sustainability. A literature review showed that no work has directly linked these issues.

Few studies that integrate operational research and sustainable operations can be found in the literature. Nouira et al. (2014) show that the optimization models for manufacturing systems should evolve to consider the environmental impacts of manufacturing activities and to integrate the environmental performance of finished products. Two optimization models that integrate environmental legislation were developed to accompany the selection of production processes. In both models, the greenness of the output product is a decision variable that is simultaneously determined along with other decisions.

Kantas et al. (2015) developed a mixed-integer linear programming capacitated lot-sizing model for analyzing the economic and environmental feasibility of ethanol production using multiple biomass sources. The model minimizes the cost of ethanol production while penalizing its possible adverse environmental impacts such as $\mathrm{CO}_{2}$ emissions and excessive water usage. The results show that the model provides insights and strategies to achieve sustainable and economically viable biofuel production using various biomass types. Su et al. (2017) propose an approach that combines carbon footprint analysis with the advantages of random search and evolutionary computing to solve the production planning problem and to reduce the carbon emissions of the enterprise.

Despite some integrations between operational problems and sustainable operations, there are no studies integrating cutting stock problems and sustainability. Thus, in this paper we propose a variant of the one-dimensional CSPUL. From now one, we consider that retails in stock can be either used internally or sold to other companies that can use them as raw material. This problem is called the cutting stock problem using or selling retails (CSPUSR). We propose a new mathematical model for the CSPUSR whose objective is to maximize the profit from cut objects and retails and, if more attractive, sell them to other companies. Furthermore, the length and number of retails are predefined and cannot exceed the maximum amount for each type. To solve the problem, we used the column generation technique (Gilmore and Gomory, 1963). In addition, two heuristic procedures are proposed to obtain integer solutions that take into account the use of retails. Computational tests were performed with randomly generated instances, simulating periods of time in order to evaluate the performance of the proposed strategy and discuss the positive implications of the solutions considering the dimensions of sustainability theory.

Drawing on the win-win approach to sustainable management (Elkington, 1994; Van der Byl and Slawinski, 2015), this study also contributes to the literature by proposing a bridge between the CSPUSR and sustainability. Although these problems are related, there is no research that addresses both simultaneously. Based on the frameworks of cleaner production $(\mathrm{CP})$ and eco-efficiency (Govindan et al., 2014), we discuss the contributions of the proposed model to improve economic, environmental and social outputs. One way to think about the planning and structure of ecoefficiency is by considering the concepts of $\mathrm{CP}$, known as an integrated, preventive environmental strategy. It can be applied to processes, products and services to increase eco-efficiency reducing risks to humans and the environment (Khalili et al., 2015) and it requires changing organizational attitudes. A CP strategy may also be considered a competitive advantage for companies (Van Hoof and Lyon, 2013). Therefore, based on previous assumptions from the literature, this study (Jaehn, 2016) explores the potential contributions of the proposed model to sustainability according to the following benefits:

- In terms of environmental benefits, the proposed model offers companies a more efficient way of cutting raw materials and the possibility of using retails, reducing possible waste that would be discarded. 
- In terms of economic benefits, the proposed model optimizes the cutting process, avoiding excessive waste. Consequently, companies' financial performance tends to improve. Companies can also sell the waste, creating opportunities for investment recovery.

- In terms of social benefits, better environmental performance of companies contributes to a better quality of life for the population. In addition, better economic performance tends to generate more jobs and better salaries.

From our point of view, the model is ideal for smaller-sized companies. As reported in the literature, smaller companies often face many barriers and deserve customized models for their specific characteristics (Freel, 2000; Walker et al., 2014).

Finally, the main contributions of this paper to some of the most relevant and contemporary topics on sustainable operations (Jaehn, 2016; Dubey et al., 2015, 2017; Kusi-Sarpong, 2016) are: 1) a mathematical model proposed to represent the cutting stock problem using or selling retail items (CSPUSR); 2) two heuristic procedures proposed to obtain integer solutions that take into account the use of retail items; 3 ) integration between the CSPUSR and sustainability; 4) discussion of the contributions of the proposed model to improve economic, environmental and social outputs.

This paper is organized as follows: in the next section, we define the problem and the proposed mathematical model to solve the one-dimensional CSPUSR. Two residual heuristic procedures to obtain integer solutions are described in the third section. The computational test results are presented in the fourth section. The fifth section discusses the results considering the dimensions of sustainability. Finally, in the final section the conclusions of this study are presented.

\section{Mathematical model for the CSPUSR}

In the CSPUSR, a set of demanded items must be produced by cutting either standard objects or retails. Similarly to the CSP, the demands for each item and the number of objects in stock are known. The demands must be met exactly by cutting the available objects and retails in order to maximize the profit. Limited quantities of each type of predefined retail can be generated for stock without being counted as a waste. Retails in stock can be used during the cutting process or, if more profitable, sold to other companies that cut these objects to meet their consumer requests.

We propose a mathematical model for the CSPUSR, which was based on the mathematical model presented in Arenales et al. (2015). To model the CSPUSR, we use the following notation:

Constants and Sets:

- $S$ : number of types of standard objects. We denote the type of an object by $s, s \in\{1, \ldots, S\}$;

- $R$ : number of types of retails. We denote a retail by $k, k \in\{1, \ldots$, $R\}$;

- $L_{s}$ : length of the standard object $s, s=1, \ldots, S$;

- $L r_{k}$ : length of the retail $k, k=1, \ldots, R$;

- $e_{s}$ : number of standard objects of type $s$ in stock, $s=1, \ldots, S$;

- er $_{k}$ : number of retails of type $k$ in stock, $k=1, \ldots, R$;

- $m$ : number of type of demanded items;

- $l_{i}$ : length of item type $i, i=1, \ldots, m$;

- $d_{i}$ : demand for item type $i, i=1, \ldots, m$;

- $J_{s}$ : set of cutting patterns for standard object type $s, s=1, \ldots, S$. In these cutting patterns, it is not possible to generate retails;

- $J r_{k}$ : set of cutting patterns for retail type $k$ in stock, $k=1, \ldots, R$;

- $J_{s}(k)$ : set of cutting patterns for the standard objects of type $s$, that generate a retail type $k, k=1, \ldots, R, s=1, \ldots, S$;
- $a_{i j s}$ : number of items type $i$ in cutting pattern $j$ for object type $s$, $i=1, \ldots, m, s=1, \ldots, S, j \in J_{s}$;

- $a_{i j s k}$ : number of items type $i$ in cutting pattern $j$ for object type $s$ and generating a retail type $k, i=1, \ldots, m, k=1, \ldots, R, s=1, \ldots, S, j$ $\in J_{s}(k)$;

- $a r_{i j k}$ : number of items type $i$ in cutting pattern $j$ for retail type $k$, $i=1, \ldots, m, k=1, \ldots R, j \in J r_{k}$;

- $c_{j s}$ : profit by cutting an object type $s$ using the cutting pattern $j$, $s=1, \ldots, S, j \in J_{s}$;

- $c_{j k}$ : profit by cutting an object type $s$ using the cutting pattern $j$ and generating a retail type $k, s=1, \ldots, S, k=1, \ldots, R, j \in J_{s}(k)$;

- $c r_{j k}$ : profit by cutting a retail type $k$ using the cutting pattern $j$, $k=1, \ldots, R, j \in J r_{k}$

- $v_{k}$ : selling price for a retail type $k, k=1, \ldots, R$;

- $U_{k}$ : maximum number of retails of type $k$ that can be generated, $k=1, \ldots, R$. At the end of the process, the stock will have at most $U_{k}$ retails of each type;

- Variables:

- $x_{j s}$ : number of objects type $s$ used in the cutting pattern $j, s=1$, $\ldots, S, j \in J_{s}$

- $x_{j s k}$ : number of objects type $s$ used in the cutting pattern $j$ and generating a retail type $k, s=1, \ldots, S, k=1, \ldots, R, j \in J_{s}(k)$;

- $x r_{j k}$ : number of retails type $k$ used in the cutting pattern $j, k=1$, $\ldots, R, j \in J r_{k}$;

- $y_{k}$ : number of retails of type $k$ sold, $k=1, \ldots, R$.

\section{Mathematical Model:}

$$
\begin{aligned}
\operatorname{Maximize} f(\mathrm{x})= & \sum_{S=1}^{S} \sum_{j \in J_{s}} c_{j s} x_{j s}+\sum_{S=1}^{S} \sum_{k=1}^{R} \sum_{j \in J_{s}(k)} c_{j s k} x_{j s k}+\sum_{k=1}^{R} \\
& \times \sum_{j \in J r_{k}} c r_{j k} x r_{j k}+\sum_{k=1}^{R} v_{k} y_{k}
\end{aligned}
$$

Subject to:

$$
\begin{aligned}
& \sum_{S=1}^{S} \sum_{j \in J_{s}} a_{i j s} x_{j s}+\sum_{s=1}^{S} \sum_{k=1}^{R} \sum_{j \in J_{s}(k)} a_{i j s k} x_{j s k} \\
& \quad+\sum_{k=1}^{R} \sum_{j \in J r_{k}} a r_{i j k} x r_{j k}=d_{i}, \quad i=1, \ldots, m \\
& \sum_{j \in J_{s}} x_{j s}+\sum_{k=1}^{R} \sum_{j \in J_{s}(k)} x_{j s k} \leq e_{S}, \quad s=1, \ldots, S \\
& \sum_{j \in J r_{k}} x r_{j k}+y_{k} \leq e r_{k}, \quad k=1, \ldots, R
\end{aligned}
$$

$\sum_{S=1}^{S} \sum_{j \in J_{s}(k)} x_{j s k}-\sum_{j \in J r_{k}} x r_{j k}-y_{k} \leq U_{k}-e r_{k} \quad k=1, \ldots, R$

$x_{j s} \geq 0, s=1, \ldots, S, j \in J_{\mathrm{s}}$ and integer

$x_{j s k} \geq 0, s=1, \ldots, S, k=1, \ldots, R, j \in J_{S}(k)$ and integer

$x r_{j k} \geq 0, k=1, \ldots, R, j \in J r_{\mathrm{k}}$ and integer

$y_{k} \geq 0, k=1, \ldots, R, \quad$ and integer

In the model (1) - (6), the main decision that must be made is to determine how often each cutting pattern must be used. Objective function (1) aims to maximize the profit obtained by cutting standard objects, as well as cutting and selling retails. In order to calculate constants $c_{j s}, c_{j s k}$, and $c r_{j k}$, which represent the profit 
obtained by each cutting pattern, we consider that the objects available in stock (standard objects or retails) and the items requested by consumers have an associated value. Let $V_{s}$ be the value of each object type $s$ in stock, $s=1, \ldots, S$, and $v_{i}$ the selling value of each item of type $i, i=1, \ldots, m$. The profit of a cutting pattern $j$ for a standard object $s$ is calculated as $\sum_{i=1}^{m}\left(v_{i} * a_{i j s}\right)-V_{s}$. Similarly, the profit of a cutting pattern for a retail can be obtained, but with an associated value for each retail type $k$ in stock, $k=1, \ldots$, $R$.

Constraint (2) ensures that the number of cut items meets the demand. Constraint (3) ensures that the quantity of standard objects used during the cutting process will not exceed the quantity available in stock. Constraint (4) ensures that the amount of each type of retail used during the cutting process, either for cutting or for selling, is not higher than the amount of the respective type of retail available in stock. Constraint (5) establishes an upper bound on the quantity of each type of retail that is allowed at the end of the cutting process. Finally, constraint (6) enforces the integrality and non-negativity of the variables.

Based on the description of the mathematical model, from our point of view, the model has implications for sustainable management in terms of environmental issues (better use of raw material and promoting the re-use of materials based on cleaner production), economic issues (optimized profits), and social issues (the model is ideal for smaller companies responsible for creating many jobs).

According to the model (1)-(6), a standard object can be completely or partially cut. In the latter, two objects are generated: a reduced object, which will be cut into items, and a retail that will be returned to stock to meet future demands or, if more profitable, sold to other companies. Fig. 1 shows a standard object with a partial cut.

In this paper, we only consider retails from the stock that can be sold. Another possibility would be to generate retails to sell (without returning them to the stock). This possibility does not change the model (1)-(6) as we only consider that retails are items demanded during the cutting process. Cui and Yang (2010) used this approach with a different model and ruled out the possibility of selling the retails.

Due to the large number of variables and the integrality constraints, it is very difficult to solve the model (1)-(6) until optimality. For this reason, the integrality conditions (6) are relaxed and the column generation technique (Gilmore and Gomory, 1963) is used making some changes to consider the possibility of generating and selling retails. Arenales et al. (2015) described in detail the changes to solve their problem. Our changes are similar to those proposed by Arenales et al. (2015) with differences in the objective function of the problem and by considering retails individually.

Because the solutions obtained by the relaxed model are not integers, heuristic procedures were also proposed and will be presented next.

\section{Heuristic procedures}

The Simplex Method using the Column Generation, when applied to the model (1)-(6) with relaxed integrality constraints, provides a continuous optimal solution for the CSPUSR. In this section, we propose two residual heuristic procedures to generate integer solutions for the problem.
Residual heuristics consist of solving the CSP with relaxed integrality conditions and, from the continuous solution, obtaining an approximate integer solution. When this procedure is adopted, a residual demand can be generated leading to a residual problem that is also solved with the relaxed integrality constraints. Therefore, an approximate integer solution for the residual problem can be obtained. This process is repeated successively until the residual demand or the approximate integer solution of the residual problem is null. Poldi and Arenales (2009) provided details of residual heuristics.

The heuristics presented hereafter consist of obtaining a good quality integer solution for the CSPUSR. Such heuristics also consider the usable leftovers. Let $j=1, \ldots, n$ be the quantity of cutting patterns in a continuous solution, obtained by solving the model (1)-(6) with relaxed integrality constraints and $j \in J_{s} \cup J r_{k} \cup J_{s}(k)$. Furthermore, let $h_{j}$ be the type of object (standard or retail) associated with the cutting pattern $j$ in a continuous solution. We define $g_{j h_{j}}$ as the profit obtained by cutting an object $h_{j}$ using the cutting pattern $j, j=1, \ldots, n$ and $p_{j h_{j}}$ as the waste when object $h_{j}$ is cut using the cutting pattern $j, j=1, \ldots, n$.

The proposed heuristics are called Residual 1 and Residual 2. For these heuristics, we solve the model (1)-(6) with the relaxed integrality conditions by the simplex method with column generation to obtain a continuous optimal solution $\bar{x}$. If this solution is not an integer, an approximate integer solution $\bar{y}$ is obtained as follows:

\subsection{Residual heuristic 1}

Order the cutting patterns according to their waste:

$p_{1 h_{1}} \leq p_{2 h_{2}} \leq \ldots \leq p_{n h_{n}}$

For each pattern, if $p_{j h_{j}} \leq \delta L_{s}$ or $p_{j h_{j}} \leq \delta L r_{k}$ (i.e., the waste is less than or equal to a percentage $\delta$ of the length of the cut object), $s=1, \ldots, S, k=1, \ldots, R$, then the integer frequency of this pattern is obtained as follows:

$\bar{y}_{j h_{j}} \leftarrow\left\lfloor\bar{x}_{j h_{j}}\right\rfloor+1$

where $\lfloor x\rfloor$ is the largest integer less than or equal to $x$. Otherwise:

$\bar{y}_{j h_{j}} \leftarrow\left\lfloor\bar{x}_{j h_{j}}\right\rfloor$ by:

With this procedure, an approximate integer solution is given

$\bar{y}=\left(\bar{y}_{1 h_{1}}, \bar{y}_{2 h_{2}}, \ldots, \bar{y}_{n h_{n}}\right)$

The next step checks the feasibility of the integer solution. We consider $\bar{y}=\left(\bar{y}_{1 h_{1}}, 0, \ldots, 0\right)$ and verify whether the demand and stock constraints are violated (constraints (2)-(4) of the model (1)-(6)). When there is an excess of items or excessive use of objects in stock, we do $\bar{y}_{1 h_{1}} \leftarrow \bar{y}_{1 h_{1}}-1$. Once the feasibility of the first cutting pattern is verified, the value is fixed and the demand and stock are updated. Then, the next cutting pattern is analyzed, i.e., we consider $\bar{y}=\left(\bar{y}_{1 h_{1}}, \bar{y}_{2 h_{2}}, 0, \ldots, 0\right)$. This procedure is repeated for all cutting patterns. If the frequency of a cutting pattern becomes null, it is discarded.

\section{Standard object}


In order to consider the retails, after the rounding procedure has been done, the cutting patterns that generated retails are analyzed again. If constraint (5) is violated, i.e., the number of generated retails exceeds the limit, considering the way these patterns were sorted, the frequency of the first pattern cutting that contains a retail is reduced by one until it becomes feasible. Demand, stock, and retails are updated. This procedure is repeated for all cutting patterns that generate retails. If the frequency of a cutting pattern becomes null, it is discarded. This procedure generates a residual demand and this residual problem is solved in the same way.

Considering this procedure, the residual demand tends to reduce during the iterations. Furthermore, in a finite number of steps, the residual demand becomes null. Due to solution rounding, the last residual problem usually has a very low demand, generating a leftover such that $p_{j h_{j}}>\delta L_{s}$ or $p_{j h_{j}}>\delta L r_{k}, S=1, \ldots, S, k=1, \ldots$, $R$. In this case, to meet the demand, the frequency of this pattern is rounded by (8).

In order to improve the performance of the heuristic procedure, as the leftover in the last cutting pattern is generally long, it is stored as long as its length is equal or longer than the smallest retail. When this leftover (retail) is available in stock, the procedure ensures that it is the first object to be cut.

\subsection{Residual heuristic 2}

Sort the cutting patterns according to their profit:

$g_{1 h_{1}} \geq g_{2 h_{2}} \geq \ldots \geq g_{n h_{n}}$

The procedure for rounding the frequencies of the patterns and checking the feasibility is done as in Residual Heuristic 1.

In the following section, we present the results of the computational tests performed to evaluate the efficiency of the proposed strategies and discuss the contributions of the model considering sustainable issues.

\section{Computational tests}

To analyze the performance of the proposed procedures and make an analysis considering sustainability by a win-win approach (Jaehn, 2016), we made a simulation with ten periods of time (Cherri et al., 2013). In each period, information about the retails that were generated was transferred to the next period. In each period, different demands were generated and retails available in stock could be cut or sold for other companies. Using these tests, we attempted to simulate the daily production of a company. Due to the approach of this problem, this is not a multiperiod problem. For this kind of problem, see Poldi and Arenales (2010).

The Model (1)-(6) and the heuristic procedures were implemented using the OPL interface of the software CPLEX 12.5. The experiments were executed using an Intel Core i5, $1.80 \mathrm{GHz}, 4 \mathrm{~GB}$ RAM computer. The data used in the tests are presented next.

\subsection{Problem data}

- Types of standard objects: $s=1$;

- Types of retails: $k=4$;

- Types of items: $m=15$;

- Availability of the standard objects: sufficient to meet the item demand of the period;

- Length of the standard objects: $L_{1}=1200$;

- Length of the retails in stock and for stocking: $400,500,600$, and 650;

- Length of the items: the lengths $l_{i}$ of the items were generated considering three size categories: small (S), medium (M) and large (L). For each category, the length was generated in the following intervals:

- Small items (S): [50,140];

- Medium items (M): [140,350]:

- Large items (L): [350,750].

- Demand: the item demand was generated considering three categories: low (L), medium (M), and high (H). For each category, the demand was generated in the following intervals:

- Low demand (L): [1,10];

- Medium demand (M): [10,80];

- High demand $(\mathrm{H})$ : $[80,300]$.

- Selling value of retails in stock: $v_{k}=1.1 L r_{k}, k=1, \ldots, 4$;

- Selling value of the items: $1.25 \ell_{i}, i=1, \ldots, m$;

- Maximum number of generated retails of each type: $U_{k}=0$, $U_{k}=2, U_{k}=4, U_{k}=6$. That is, when $U_{k}=2$, at most two retails of each type $k, k=1,2,3,4$ can be generated to stock per period;

- Parameter: $=0.1$.

Combining the length and demand categories, nine instance classes were randomly generated. For example, the class $[\mathrm{LH}]$ represents a class with large items and high demands. These are the values that we have established for the tests, but other values also could have been used.

Table 1 shows the average profit for the 10 periods. In all classes, in the first period the stock only consists of standard objects, that is, $e r_{k}=0, k=1, \ldots, 4$. In these tests, the solution for the classical cutting stock problem $\left(U_{k}=0 e r_{k}=0, k=1, \ldots, 4\right)$ can be analyzed. From the second period, the retails generated in the first period will be in stock and can be cut or sold in the next one. In this table, when $U_{\mathrm{k}}=0, k=1,2,3,4$, retail should not be generated in any period. When $U_{\mathrm{k}}=2, k=1,2,3,4$, up to two retails of each type in each period can be generated and so on.

The table shows that there is more profit for higher values of $U_{\mathrm{k}}$, $k=1,2,3,4$. This increase in profit was expected because the retails increased the variety of objects in stock, improving the quality of the cutting patterns. In addition, retails in stock can be sold, increasing the company's profit. It can be observed that in some classes of medium and small items, the quality of the solution did not increase to higher values of $U_{\mathrm{k}}$. This occurred due to rounding done by the heuristics.

Table 2 presents the medium profit obtained from cutting and selling the retails for Residual Heuristics 1 and 2. These values should be different for other selling values of retails and items.

As a natural consequence, for $U_{k}=6$ the profit obtained from cutting or selling the retails is generally larger compared with $U_{\mathrm{k}}=2$ or $U_{\mathrm{k}}=4$. As the generated retails are well managed, this possibility can improve the quality of the cutting patterns and yield extra profits for companies. If this possibility were not considered, leftover material could be discarded.

Table 3 presents the average waste generated considering the 10 periods for all classes. As seen previously, when $U_{\mathrm{k}}=0, k=1,2,3,4$, retail should not be generated in any period. When $U_{\mathrm{k}}=2, k=1,2$, 3,4 , up to two retails of each type in each period can be generated and so on.

Although minimizing the waste is not the objective of this work, we present these values because it is a natural consequence of the quality of the cutting patterns. In Table 3, it can be observed that the waste is less for greater values of $U_{k}$. This shows that the strategy for generating retails significantly improves the solutions for reducing the material that should be discarded. The solution for $U_{k}=0$ is the worst since there is no retail in stock and none can be generated (classical CSP). In some exceptional cases, increasing the value of $U_{k}$ may lead to more waste. As already explained, this occurs due to the rounding procedure of the heuristics.

Regarding the computational time, the average time to solve each problem was $1.27 \mathrm{~s}$, which is very acceptable in practice. 
Table 1

Average profit.

\begin{tabular}{|c|c|c|c|c|c|}
\hline Class (Item/Demand) & Solution type & $U_{k}=0$ & $U_{k}=2$ & $U_{k}=4$ & $U_{k}=6$ \\
\hline \multirow[t]{2}{*}[\mathrm{LH}]{} & Residual 1 & $229,457.25$ & $230,792.75$ & $231,398.75$ & $232,124.75$ \\
\hline & Residual 2 & $229,457.25$ & $230,792.75$ & $231,398.75$ & $232,124.75$ \\
\hline \multirow[t]{2}{*}[\mathrm{LM}]{} & Residual 1 & $56,560.75$ & $57,550.65$ & $57,873.65$ & $58,179.65$ \\
\hline & Residual 2 & $56,560.75$ & $57,544.65$ & $57,873.65$ & $58,179.65$ \\
\hline \multirow[t]{2}{*}{ [LL] } & Residual 1 & 5493.25 & 6879.65 & 7450.75 & 7760.75 \\
\hline & Residual 2 & 5493.25 & 6879.65 & 7450.75 & 7760.75 \\
\hline \multirow[t]{2}{*}[\mathrm{MH}]{} & Residual 1 & $181,525.50$ & $182,020.00$ & $182,028.50$ & $182,071.80$ \\
\hline & Residual 2 & $181,525.50$ & $181,974.50$ & $182,033.60$ & $182,081.50$ \\
\hline \multirow[t]{2}{*}{ [MM] } & Residual 1 & $43,856.63$ & $44,619.25$ & $44,643.75$ & $44,761.25$ \\
\hline & Residual 2 & $43,992.25$ & $44,647.25$ & $44,704.25$ & $44,760.25$ \\
\hline \multirow[t]{2}{*}[\mathrm{ML}]{} & Residual 1 & 4673.63 & 5206.63 & 5217.63 & 5307.49 \\
\hline & Residual 2 & 4673.63 & 5156.63 & 5302.13 & 5263.63 \\
\hline \multirow[t]{2}{*}[\mathrm{SH}]{} & Residual 1 & $71,666.38$ & $72,444.27$ & $72,429.48$ & $72,431.88$ \\
\hline & Residual 2 & $71,666.38$ & $72,484.88$ & $72,522.88$ & $72,505.18$ \\
\hline \multirow[t]{2}{*}{ [SM] } & Residual 1 & $15,506.00$ & $15,896.10$ & $15,914.20$ & $15,896.10$ \\
\hline & Residual 2 & $15,506.00$ & $15,956.40$ & $15,929.70$ & $15,972.10$ \\
\hline \multirow[t]{2}{*}[\mathrm{SL}]{} & Residual 1 & 1265.75 & 1941.15 & 1941.15 & 1883.05 \\
\hline & Residual 2 & 1265.75 & 1924.05 & 1924.05 & 1898.05 \\
\hline
\end{tabular}

Table 2

Profit obtained from cutting and selling the retails.

\begin{tabular}{|c|c|c|c|c|c|c|c|}
\hline \multirow[t]{2}{*}{ Class (Item/Demand) } & \multirow[t]{2}{*}{ Solution Type } & \multicolumn{2}{|l|}{$U_{k}=2$} & \multicolumn{2}{|l|}{$U_{k}=4$} & \multicolumn{2}{|l|}{$U_{k}=6$} \\
\hline & & cut & sell & cut & sell & cut & sell \\
\hline \multirow{2}{*}{ [LH] } & Residual 1 & 215.78 & 32.00 & 365.77 & 58.00 & 532.90 & 84.00 \\
\hline & Residual 2 & 215.78 & 32.00 & 365.77 & 58.00 & 365.77 & 84.00 \\
\hline \multirow[t]{2}{*}{ [LM] } & Residual 1 & 85.20 & 61.50 & 108.20 & 104.50 & 135.07 & 140.50 \\
\hline & Residual 2 & 91.57 & 55.50 & 108.20 & 104.50 & 108.20 & 140.50 \\
\hline \multirow[t]{2}{*}{ [LL] } & Residual 1 & 193.60 & 10.00 & 281.78 & 20.00 & 337.58 & 30.00 \\
\hline & Residual 2 & 193.60 & 10.00 & 281.78 & 20.00 & 281.78 & 30.00 \\
\hline \multirow[t]{2}{*}[\mathrm{MH}]{} & Residual 1 & 182.12 & 49.50 & 226.00 & 108.00 & 222.25 & 133.00 \\
\hline & Residual 2 & 89.75 & 49.00 & 108.50 & 132.50 & 108.50 & 96.00 \\
\hline \multirow[t]{2}{*}{ [MM] } & Residual 1 & 59.37 & 72.00 & 116.62 & 126.50 & 49.62 & 194.00 \\
\hline & Residual 2 & 75.50 & 35.00 & 43.00 & 92.00 & 43.00 & 173.00 \\
\hline \multirow[t]{2}{*}[\mathrm{ML}]{} & Residual 1 & 165.87 & 48.00 & 160.37 & 124.00 & 185.25 & 140.50 \\
\hline & Residual 2 & 97.62 & 33.00 & 75.65 & 113.50 & 75.65 & 110.00 \\
\hline \multirow[t]{2}{*}[\mathrm{SH}]{} & Residual 1 & 104.37 & 67.00 & 120.50 & 36.50 & 133.00 & 25.00 \\
\hline & Residual 2 & 40.37 & 84.50 & 18.75 & 82.50 & 18.75 & 100.50 \\
\hline \multirow[t]{2}{*}{ [SM] } & Residual 1 & 72.37 & 93.00 & 92.50 & 101.00 & 100.00 & 75.00 \\
\hline & Residual 2 & 38.25 & 72.50 & 52.00 & 79.50 & 52.00 & 84.00 \\
\hline \multirow[t]{2}{*}[\mathrm{SL}]{} & Residual 1 & 16.25 & 96.00 & 16.25 & 96.00 & 32.50 & 71.50 \\
\hline & Residual 2 & 31.25 & 79.50 & 31.25 & 79.50 & 31.25 & 53.50 \\
\hline
\end{tabular}

Table 3

Average waste.

\begin{tabular}{|c|c|c|c|c|c|}
\hline Class (Item/Demand) & Solution Type & $U_{k}=0$ & $U_{k}=2$ & $U_{k}=4$ & $U_{k}=6$ \\
\hline \multirow[t]{2}{*}[\mathrm{LH}]{} & Residual 1 & $174,418.20$ & $173,114.70$ & $172,534.70$ & $171,834.70$ \\
\hline & Residual 2 & $174,418.20$ & $173,114.70$ & $172,534.70$ & $171,834.70$ \\
\hline \multirow[t]{2}{*}{ [LM] } & Residual 1 & $35,823.40$ & $34,895.00$ & $34,615.00$ & $34,433.40$ \\
\hline & Residual 2 & $35,823.40$ & $34,895.00$ & $34,615.00$ & $34,345.00$ \\
\hline \multirow[t]{2}{*}{ [LL] } & Residual 1 & 5157.40 & 3781.00 & 3219.90 & 2919.90 \\
\hline & Residual 2 & 5157.40 & 3781.00 & 3219.90 & 2919.90 \\
\hline \multirow[t]{2}{*}[\mathrm{MH}]{} & Residual 1 & 675.60 & 230.60 & 280.60 & 262.30 \\
\hline & Residual 2 & 675.60 & 275.60 & 300.00 & 215.60 \\
\hline \multirow[t]{2}{*}[\mathrm{MM}]{} & Residual 1 & 746.70 & 223.20 & 353.20 & 303.20 \\
\hline & Residual 2 & 878.20 & 258.20 & 258.20 & 283.20 \\
\hline \multirow[t]{2}{*}[\mathrm{ML}]{} & Residual 1 & 653.10 & 168.10 & 233.10 & 193.10 \\
\hline & Residual 2 & 653.10 & 203.10 & 138.10 & 173.10 \\
\hline \multirow[t]{2}{*}[\mathrm{SH}]{} & Residual 1 & 866.90 & 156.00 & 140.30 & 126.40 \\
\hline & Residual 2 & 866.90 & 132.90 & 92.90 & 128.60 \\
\hline \multirow[t]{2}{*}[\mathrm{SM}]{} & Residual 1 & 459.20 & 162.10 & 152.00 & 144.10 \\
\hline & Residual 2 & 459.20 & 81.30 & 115.00 & 77.10 \\
\hline \multirow[t]{2}{*}[\mathrm{SL}]{} & Residual 1 & 691.40 & 112.00 & 112.00 & 145.60 \\
\hline & Residual 2 & 691.40 & 112.60 & 112.60 & 112.60 \\
\hline
\end{tabular}




\section{Discussion}

In order to have better integration between operational research and sustainability dimensions (environmental, economic and social), this study addresses the one-dimensional cutting stock problem by using or selling retails (CSPUSR) considering the potential of an emerging theory: sustainable operations (Jaehn, 2016). Despite of an emerging theory, this is an important contribution to the literature because it is the first study that relates a cutting problem with its implications for sustainability.

In this kind of operational problem, retails in stock can be used to meet demands or, if more attractive, they can be sold to other companies. Originally, we considered potential contributions of the proposed model for the sustainability dimensions from the perspective of a win-win approach, highlighting environmental contributions, economic gains, and social issues. Thus, our approach is a response to the call for more integration between operation research applications and sustainability (Brandenburg et al., 2014; Govindan et al., 2014; Gunasekaran and Irani, 2014; Jaehn, 2016).

The first contribution of this study to the literature is to propose a new mathematical model with the aim of maximizing the profit obtained by cutting or selling retails. In addition, the size and number of retails generated during the cutting process are previously defined and limited for each type of retail. The mathematical model was solved using the simplex method with column generation and, in order to obtain integer solutions, two heuristic procedures that consider using leftovers were also proposed.

The performance of the proposed strategy was evaluated by simulating a situation by the period of time. Considering several aspects, the experiments showed clearly that producing retails to be used in the future to cut items or to be sold yields better profits compared to a situation where retails cannot be generated. Generating retails increases the diversity of objects in stock, and consequently, the quality of the cutting patterns. Considering the solutions obtained in this paper, we believe that the CSPUSR can be used as an alternative by some companies that cut objects in their production line. In this study, the strategy was applied to onedimensional cutting problems, but it can easily be extended and applied to two-dimensional cutting problems.

Based on the available literature on the subject, this work contributes a new mathematical model to represent a problem not studied and relate its decisions with implication for sustainability. This research adds evidence to the literature by exemplifying the win-win approach to sustainable management (Elkington, 1994; Van der Byl and Slawinski, 2015). The work can be view as an original a bridge between the CSPUSR and sustainable operations. Althought CSPUSR and sustainable operations are clearly related, there are no enough research that addresses both simultaneously. Based on the frameworks of cleaner production (CP) and ecoefficiency (Govindan et al., 2014), we discuss the contributions of the proposed model to improve economic, environmental and social outputs. One way to think about the planning and structure of eco-efficiency is by considering the concepts of $\mathrm{CP}$, known as an integrated, preventive environmental strategy. It can be applied to processes, products and services to increase eco-efficiency reducing risks to humans and the environment (Khalili et al., 2015) and it requires changing organizational attitudes. A CP strategy may also be considered a competitive advantage for companies (Van Hoof and Lyon, 2013). Therefore, based on previous assumptions from the literature (Jaehn, 2016), this study adds evidence of the positive and mutually beneficial integration between operational research and sustainability, towards sustainable manufacturing.

In terms of sustainability, the proposed model for the CSPUSR is relevant because it would contribute to:

- Improving economic performance of companies by increasing the profit. The possibility of generating and selling retail can be an alternative to companies reducing their residues and increasing profits, as shown in Table 1.

- Adopting a more eco-efficient approach based on a cleaner production perspective, reducing waste and promoting the use of retails. As shown in Table 2, in most cases, the profit from cutting and using retail internally in a company is higher than that of selling it. This characteristic of the model is aligned to the cleaner production perspective (Van Hoof and Lyon, 2013). According to the cleaner production perspective, reducing waste generation or using retail internally is better than sending it out to other partners across a supply chain (Khalili et al., 2015). This is because using retails internally avoids potential environmental impacts that would be generated when selling, transporting and moving retails to other companies;

- Improving social aspects. First, the model is ideally customized to the reality of smaller companies. As smaller companies tend to face many barriers in the current economy, new managerial models and tools that would help managers of small-sized companies are always welcome (Freel, 2000). Additionally, as the model is able to generate retail, it would be useful for other small companies in the supply chain (Zhu et al., 2008), thus boosting the local economy and creating jobs.

Finally, the model shows how operational research models can contribute directly to a more circular economy (Brandenburg et al., 2014), in which retail becomes raw materials for other production processes, towards more eco-efficient (Govindan et al., 2014) and sustainable operations management (Jaehn, 2016; Dubey et al., 2015, 2017; Kusi-Sarpong et al., 2016).

\section{Conclusion}

This paper proposes a mathematical model and two heuristic procedures to solve the cutting stock problem with usable leftovers and selling retails. It also discusses the implications for sustainability, analyzing the economic, environmental and social issues. This link between classical problems of operational research and sustainability has been proposed by researchers, however based on the knowledge of the authors, this is the first study that relates a cutting problem with its implications for sustainability. Moreover, it addresses a gap in the literature.

Furthermore, this paper shows how mathematical approaches in operational research can be used to support a win-win approach in sustainable management, as proposed by Brandenburg et al. (2014).

\section{Acknowledgment}

The authors would like to thank the Fundação de Amparo a Pesquisa do Estado de São Paulo (FAPESP - Processo: 15/03066-8); and the Conselho Nacional de Desenvolvimento Científico e Tecnológico (CNPq - Bolsista CNPq and Processo: 477481/2013-2) for the financial support.

\section{References}

Abuabara, A., Morabito, R., 2009. Cutting optimization of structural tubes to build agricultural light aircrafts. Ann. Oper. Res. 169 (1), 149-165.

Andrade, R, Birgin, EG, Morabito, R, Ronconi, D.P, 2013. MIP models for two-

dimensional non-guillotine cutting problems with usable leftovers. J. Oper. Res. Soc. 65, 1649-1663.

Andrade, R., Birgin, E.G., Morabito, R., 2016. Two-stage two-dimensional guillotine 
cutting stock problems with usable leftovers. Int. Trans. Oper. Res. 23, 121-145. Arenales, M.N., Cherri, A.C., Nascimento, D.N., Vianna, A.C.G., 2015. A new mathematical model for the cutting stock/leftover problem. Pesqui. Oper. 35 (3), 1-14.

Brandenburg, M., Govindan, K., Sarkis, J., Seuring, S., 2014. Quantitative models for sustainable supply chain management: developments and directions. Eur. J. Oper. Res. 233 (2), 299-312.

Cherri, A.C., Arenales, M.N., Yanasse, H.H., 2009. The one-dimensional cutting stock problem with usable leftovers - a heuristic approach. Eur. J. Oper. Res. 196 (3), 897-908.

Cherri, A.C., Arenales, M.N., Yanasse, H.H., 2013. The usable leftover onedimensional cutting stock problem-a priority-in-use heuristic. Int. Trans. Oper. Res. 20 (2), 189-199.

Cherri, A.C., Arenales, M.N., Yanasse, H.H., Poldi, K.C., Vianna, A.C.G., 2014. The onedimensional cutting stock problem with usable leftovers - a survey. Eur. J. Oper. Res. 236 (2), 395-402.

Cui, Y., Yang, Y., 2010. A heuristic for the one-dimensional cutting stock problem with usable leftovers. Eur. J. Oper. Res. 204 (2), 245-250.

Dubey, R., Gunasekaran, A., Chakrabarty, A., 2015. World-class sustainable manufacturing: framework and a performance measurement system. Int. J. Prod. Res. 53 (17), 5207-5223.

Dubey, R., Gunasekaran, A., Childe, S.J., Papadopoulos, T., Hazen, B., Giannakis, M., Roubaud, D., 2017. Examining the effect of external pressures and organizational culture on shaping performance measurement systems (PMS) for sustainability benchmarking: some empirical findings. Int. J. Prod. Econ. 193, $63-76$.

Elkington, J., 1994. Towards the sustainable corporation: win-win-win business strategies for sustainable development. Calif. Manag. Rev. 36 (2), 90-100.

Freel, M.S., 2000. Barriers to product innovation in small manufacturing firms. Int. Small Bus. J. 18 (2), 60-80.

Gilmore, P.C., Gomory, R.E., 1961. A linear programming approach to the cuttingstock problem. Oper. Res. 9 (6), 849-859.

Gilmore, P.C., Gomory, R.E., 1963. A linear programming approach to the cutting stock problem-Part II. Oper. Res. 11 (6), 863-888.

Gonzalez, E.D., Sarkis, J., Huisingh, D., Huatuco, L.H., Maculan, N., MontoyaTorres, J.R., de Almeida, C.M., 2015. Making real progress toward more sustainable societies using decision support models and tools: introduction to the special volume. J. Clean. Prod. 105, 1-13.

Govindan, K., Sarkis, J., Chiappetta Jabbour, C.J., Zhu, Q., Geng, Y., 2014. Eco-efficiency based green supply chain management: current status and opportunities. Eur. J. Oper. Res. 293-298.

Gradisar, M., Trkman, P., 2005. A combined approach to the solution to the general one-dimensional cutting stock problem. Comput. Oper. Res. 32, 1793-1807.

Gradišar, M., Jesenko, J., Resinovič, G., 1997. Optimization of roll cutting in clothing industry. Comput. Oper. Res. 24 (10), 945-953.

Gunasekaran, A., Irani, Z., 2014. Sustainable Operations Management: design, modelling and analysis. J. Oper. Res. Soc. 65 (6), 801-805.

Jaehn, F., 2016. Sustainable operations. Eur. J. Oper. Res. 253, 243-264.

Kantas, A.B., Cobuloglu, H.I., Büyüktahtakin, I.E., 2015. Multi-source capacitated lotsizing for economically viable and clean biofuel production. J. Clean. Prod. 94 $116-129$.

Kantorovich, L.V., 1960. Mathematical methods of organizing and planning production. Manag. Sci. 6 (4), 366-422.

Khalili, N.R., Duecker, S., Ashton, W., Chavez, F., 2015. From cleaner production to sustainable development: the role of academia. J. Clean. Prod. 96, 30-43.

Kusi-Sarpong, S., Sarkis, J., Wang, X., 2016. Assessing green supply chain practices in the Ghanaian mining industry: a framework and evaluation. Int. J. Prod. Econ. $181,325-341$.

Nouira, I., Frein, Y., Hadj-Alouane, A.B., 2014. Optimization of manufacturing systems under environmental considerations for a greenness-dependent demand. Int. J. Prod. Econ. 150, 188-198.

Poldi, K.C., Arenales, M.N., 2009. Heuristics for the one-dimensional cutting stock problem with limited multiple stock lengths. Comput. Oper. Res. 36 (6) 2074-2081.

Poldi, K.C., Arenales, M.N., 2010. Problema de corte de estoque multiperíodo. Pesqui. Oper. 30 (1), 153-174.

Scheithauer, G., 1991. A note on handling residual lengths. Optimization 22 (3) 461-466.

Su, Q., Yang, W., Liu, Y., 2017. Optimization of carbon emission considering production planning at enterprise level. J. Clean. Prod. 162, 635-645.

Tomat, L., Gadišar, M., 2017. One-dimensional stock cutting: optimization of usable leftovers in consecutive orders. Central Eur. J. Oper. Res. 25 (2), 473-489.

Van der Byl, C.A., Slawinski, N., 2015. Embracing tensions in corporate sustainability A review of research from win-wins and trade-offs to paradoxes and beyond. Organ. Environ. 28 (1), 54-79.

Van Hoof, B., Lyon, T.P., 2013. Cleaner production in small firms taking part in Mexico's Sustainable Supplier Program. J. Clean. Prod. 41, 270-282.

Walker, H., Seuring, S., Sarkis, J., Klassen, R., 2014. Sustainable operations management: recent trends and future directions. Int. J. Oper. Prod. Manag. 34 (5), $1-3$.

Zhu, Q., Sarkis, J., Lai, K.H., 2008. Confirmation of a measurement model for green supply chain management practices implementation. Int. J. Prod. Econ. 111 (2) $261-273$. 